\title{
Neutral Saturated Lapse Rate: An Experimental Check from CALJET-1998 and PACJET-2001
}

\author{
RENZO RICHIARDONE AND MASSIMILIANO MANFRIN \\ Dipartimento di Fisica Generale, Università di Torino, Turin, Italy
}

(Manuscript received 17 March 2009, in final form 26 June 2009)

\begin{abstract}
The lapse rates of high-resolution temperature profiles during nearly neutral, saturated conditions are compared with the saturated adiabatic lapse rate and with that proposed by Richiardone and Giusti. A good agreement between the latter and the mean value of the observed lapse rate is found, whereas the saturated adiabatic lapse rate differs significantly, confirming experimentally that it is not completely correct to assess the moist neutrality from a comparison with the saturated adiabatic lapse rate. The lapse-rate distribution supports the hypothesis that the lapse-rate statistics is a local collection of saturated adiabatic lapse rates in a background normal distribution centered around the neutrality.
\end{abstract}

\section{Introduction}

The moist Brunt-Väisälä frequency squared,

$$
\begin{aligned}
N_{w}^{2}= & \frac{g}{T}\left(1+\frac{L_{v} r_{w}}{R_{d} T}\right)\left(\frac{d T}{d z}+\Gamma_{w}\right) \\
& -\frac{g}{1+r_{t w}} \frac{d r_{w}}{d z}-\frac{g}{1+r_{t w}} \frac{d r_{l}}{d z},
\end{aligned}
$$

from Durran and Klemp (1982) is commonly used to assess if a saturated atmosphere is stable $\left(N_{w}^{2}>0\right)$, unstable $\left(N_{w}^{2}<0\right)$, or neutral $\left(N_{w}^{2}=0\right)$, Here $g$ is the gravitational acceleration, $T$ is the temperature, $L_{v}$ is the specific latent heat of vaporization (replaced by that for sublimation if the saturation is over ice), $R_{d}$ is the gas constant of dry air, $r_{w}$ is the (saturated) vapor mixing ratio, $r_{l}$ is the condensed phase (liquid or solid) mixing ratio, $r_{t w}=r_{w}+r_{l}$ is the total water mixing ratio, $z$ is the height, and $\Gamma_{w}$ is the saturated adiabatic lapse rate.

The comparison of the lapse rate $(\Gamma \equiv-d T / d z)$ is often used to assess the stability of a saturated atmosphere, but Eq. (1) indicates that that is not completely correct, as first shown by Lalas and Einaudi (1974). As a matter of fact, in Eq. (1) the sign of $N_{w}^{2}$ is not determined by $\left(d T / d z+\Gamma_{w}\right)$ when the stability is small.

Corresponding author address: Renzo Richiardone, Dipartimento di Fisica Generale, Università di Torino, via P. Giuria 1, 10125 Torino, Italy.

E-mail: richiardone@ph.unito.it
Richiardone and Giusti (2001) showed that the gradient of the saturated vapor profile in the second term on the rhs of Eq. (1) can be written in terms of the lapse rate, and Eq. (1) can then be expressed as

$$
\begin{aligned}
N_{w}^{2}= & \frac{g}{T}\left[1+\frac{L_{v} r_{w}}{R_{d} T}\left(1-\frac{\epsilon+r_{w}}{1+r_{t w}}\right)\right]\left(\frac{d T}{d z}+\Gamma_{w}^{*}\right) \\
& -\frac{g}{1+r_{t w}} \frac{d r_{l}}{d z}
\end{aligned}
$$

where $\epsilon=R_{d} / R_{v}$ is the ratio between the gas constants of dry air and water vapor and $\Gamma_{w}^{*}$ has a complicated expression, which can be approximated by

$$
\Gamma_{w 1}^{*}=\Gamma_{d} \frac{1+1.18 \frac{L_{v} r_{w}}{R_{d} T}}{1+0.93 \frac{L_{v}^{2} r_{w}}{c_{p d} R_{v} T^{2}}},
$$

where $\Gamma_{d}$ is the dry adiabatic lapse rate and $c_{p d}$ is the specific heat of the dry air at constant pressure.

As discussed in Richiardone and Giusti (2001), Eq. (2) shows that when the contribution of the condensed water mixing ratio gradient is negligible (a reasonable approximation in the lower troposphere), the sign of $N_{w}^{2}$ is determined by $\left(d T / d z+\Gamma_{w}^{*}\right)$, implying that $\Gamma_{w}^{*}$, instead of the saturated adiabatic lapse rate $\Gamma_{w}$, is the better lapse rate to assess the stability.

The California Land-Falling Jets Experiment (CALJET) and the Pacific Land-Falling Jets Experiment (PACJET) 
were conducted to investigate the role of the pre-coldfrontal low-level jet (LLJ) on U.S. West Coast heavy rainfall episodes. The LLJ is an integral part of extratropical cyclones and is characterized by warm temperatures, weak stratification, large water content, and strong low-altitude winds (e.g., Browning and Pardoe 1973). These attributes are conducive to the production of heavy rainfall through orographic forcing when the LLJ impinges the coastal mountains. Because the region of strongest water vapor transport was found to be very narrow, and yet is responsible for almost all the meridional water vapor transport at midlatitudes (Zhu and Newell 1998), this region is referred to as an "atmospheric river" (Zhu and Newell 1998; Ralph et al. 2004).

High-resolution vertical profiles of wind velocity, temperature, and water vapor from GPS dropsondes released at about $500 \mathrm{~km}$ offshore were collected from the National Oceanic and Atmospheric Administration (NOAA) P-3 aircraft in 10 storms during the CALJET of 1998 and in 7 storms during the PACJET of 2001. One sounding into the pre-cold-frontal LLJ was chosen from each storm in order to provide a set of high-resolution (roughly $10 \mathrm{~m}$ ) profiles that are representative of the thermodynamic conditions in the LLJ region ahead of a landfalling cold front. For those storms with more than one applicable profile, the sounding with the largest vertically integrated water vapor content was chosen to ensure that the sounding closest to the core of the atmospheric river was included (Ralph et al. 2005).

Ralph et al. (2005) calculated the moist Brunt-Väisälä frequency squared of the composite mean sounding using Eq. (1), and showed that the moisture-rich pre-coldfrontal LLJ is approximately neutrally stratified below $2.8 \mathrm{~km}\left(N_{w}^{2} \approx 0\right)$. As the derivation for $\Gamma_{w}^{*}$ starts from Eq. (1), the mean lapse rate of the composite profile is expected to be approximately equal to $\Gamma_{w}^{*}$ and slightly greater than the saturated adiabatic lapse rate $\Gamma_{w}$, because $\Gamma_{w}^{*}$ is greater than $\Gamma_{w}$ at all temperatures (Richiardone and Giusti 2001).

A comparison between the lapse rates of the 17 soundings and $\Gamma_{w}, \Gamma_{w}^{*}$, and the approximate value $\Gamma_{w_{1}}^{*}$ is discussed in section 2 .

\section{Analysis}

The accuracy of the above-mentioned dropsonde profiles is approximately $0.2^{\circ} \mathrm{C}$ for temperature and $5 \%$ for relative humidity, but visual inspection of the 17 profiles suggested that they possess a dry bias in the moist lower troposphere (i.e., constant offsets between temperature and dewpoint over a significant portion of a sounding). The combined effects of different sources of error can result in underestimates of relative humidity as large as
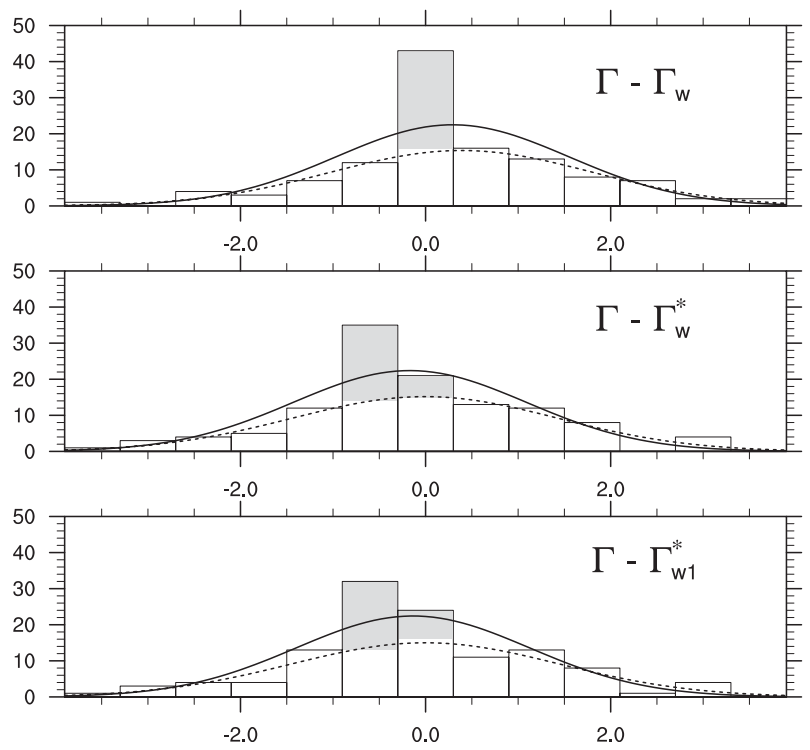

FIG. 1. Frequency distribution (118 values) of the differences between $\Gamma$ and the theoretical values: (top) $\Gamma_{w}$, (middle) $\Gamma_{w}^{*}$, and (bottom) $\Gamma_{w 1}^{*}$. The two curves represent the expected values from a normal distribution using the mean and standard deviation of the sample (continuous line) and the mean and standard deviation of the sample after having subtracted a number of values corresponding to the shaded areas (dotted line). The $x$-axis units are kelvins per kilometer.

$10 \%$ for ambient relative humidities at $\sim 90 \%$ (Ralph et al. 2005). Conservatively, only the data with a relative humidity greater than $90 \%$ will be included in the following analysis. Considering that the lapse rate will be estimated in narrow layers where the humidity is nearly constant, the dry bias can be considered constant in each layer, so that the temperature gradient is not influenced by it. Therefore, the lapse-rate analysis will not be affected by the dropsonde dry bias.

Below a height of $2.8 \mathrm{~km}$, the 17 profiles were split into 100-m layers, retaining only the layers where the relative humidity was greater than $90 \%$ and mean temperature greater than $1^{\circ} \mathrm{C}$ (to avoid any ambiguity about the liquid/solid phase). For each layer the lapse rate $\Gamma$ was then calculated by means of a linear fit, provided that it contained more than five measurements. The differences $d$ between the experimental values of $\Gamma$ and the theoretical values of $\Gamma_{w}, \Gamma_{w}^{*}$, and $\Gamma_{w 1}^{*}$ were then calculated. After having discarded one outlier by using Chauvenet's criterion (Taylor 1997), a sample of 118 elements was obtained, in a temperature range from $1^{\circ}$ to $15^{\circ} \mathrm{C}$.

After having observed that about $36 \%$ of the lapse rates fall within $0.3 \mathrm{~K} \mathrm{~km}^{-1}$ of the moist adiabatic lapse rate $\Gamma_{w}$, a bin width equal to $0.6 \mathrm{~K} \mathrm{~km}^{-1}$ has been chosen for the distribution of the differences $d$ in order to highlight this characteristic (Fig. 1). Table 1 shows the 
TABLE 1. Sample mean values and standard errors of the mean of the differences between the observed lapse rate and $\Gamma_{w}, \Gamma_{w}^{*}$, and $\Gamma_{w 1}^{*}$, with $n=118$. Units are in $\mathrm{K} \mathrm{km}^{-1}$.

\begin{tabular}{|c|c|c|c|}
\hline & $\overline{\overline{\Gamma-\Gamma_{w}}}$ & $\overline{\Gamma-\Gamma_{w}^{*}}$ & $\overline{\overline{\Gamma-\Gamma_{w 1}^{*}}}$ \\
\hline$\overline{\bar{d} \pm s_{\bar{d}}}$ & $0.29 \pm 0.12$ & $-0.17 \pm 0.12$ & $-0.14 \pm 0.12$ \\
\hline$z_{\bar{d}}=\bar{d} / s_{\bar{d}}$ & 2.42 & -1.42 & -1.17 \\
\hline$P\left(|z|>\left|z_{\bar{d}}\right|\right)$ & $1.55 \%$ & $15.56 \%$ & $24.20 \%$ \\
\hline
\end{tabular}

sample mean values $\bar{d}$ and their estimated errors $s_{\bar{d}}=s_{d} / \sqrt{n}$, where $s_{d}$ is the sample standard deviation.

It appears clearly from Fig. 1 that the central bin of the $\Gamma-\Gamma_{w}$ distribution differs significantly from the others (that a $\chi^{2}$ test showed to be consistent with a normal distribution). Notwithstanding the high percentage of values in the central bin, Table 1 shows that the mean value is positive and differs significantly from zero. If stability would be assessed by comparing the observed lapse rate with the saturated adiabatic lapse rate $\Gamma_{w}$, the CALJET-1998 and PACJET-2001 profiles would therefore be classified, in the mean, as (significantly) unstable. The sample size being greater than 50 (i.e., large enough to be considered statistically "large"; Green and Margerison 1978), the null hypothesis $\left(H_{0}\right)$ that the expected value of the sample mean is equal to zero can in fact be tested by calculating the probability $P\left(|z|>\left|z_{\bar{d}}\right|\right)$, where $z$ is a standard normal variate and $z_{\bar{d}}=\bar{d} / s_{\bar{d}}$ is derived from the sample. Table 1 shows that $H_{0}$ must be rejected at the significance level of $5 \%$, being $P\left(|z|>\left|z_{\bar{d}}\right|\right)<5 \%$.

As regards the distributions of $\Gamma-\Gamma_{w}^{*}$ and $\Gamma-\Gamma_{w 1}^{*}$, Fig. 1 shows that the highest number of bin data is partially shifted to the bin centered at $-0.6 \mathrm{~K} \mathrm{~km}^{-1}$. This is due to the offset between $\Gamma_{w}, \Gamma_{w}^{*}$, and $\Gamma_{w 1}^{*}$ : the subtraction between the mean values of the distributions gives $\overline{\Gamma_{w}^{*}}-$ $\overline{\Gamma_{w}}=0.46 \mathrm{~K} \mathrm{Km}^{-1}$ and $\overline{\Gamma_{w 1}^{*}}-\overline{\Gamma_{w}}=0.43 \mathrm{~K} \mathrm{Km}^{-1}$.

Mean values are slightly negative, implying a weak stability. However, Table 1 shows that for both distributions the null hypothesis $H_{0}$ cannot be rejected (at the significance level of $5 \%$ ), being $P\left(|z|>\left|z_{\bar{d}}\right|\right)>5 \%$.

The profiles that produce an approximately neutral $\left(N_{w}^{2} \approx 0\right)$ composite are therefore characterized, in the mean, by lapse rates that are consistent with $\Gamma_{w}^{*}$ (and its approximated value $\Gamma_{w 1}^{*}$ ). This results is not surprising, because the formulas for the moist Brunt-Väisälä frequency squared and for $\Gamma_{w}^{*}$ and $\Gamma_{w 1}^{*}$ contain the same information on stability, so the only way that they could not produce the same stability assessment would be if some mistake had been made in the derivation or analysis. On the contrary, as observed above, the profiles would be classified as significantly unstable if the saturated adiabatic lapse rate were used to assess the stability. Separate analyses of the CALJET and PACJET profiles
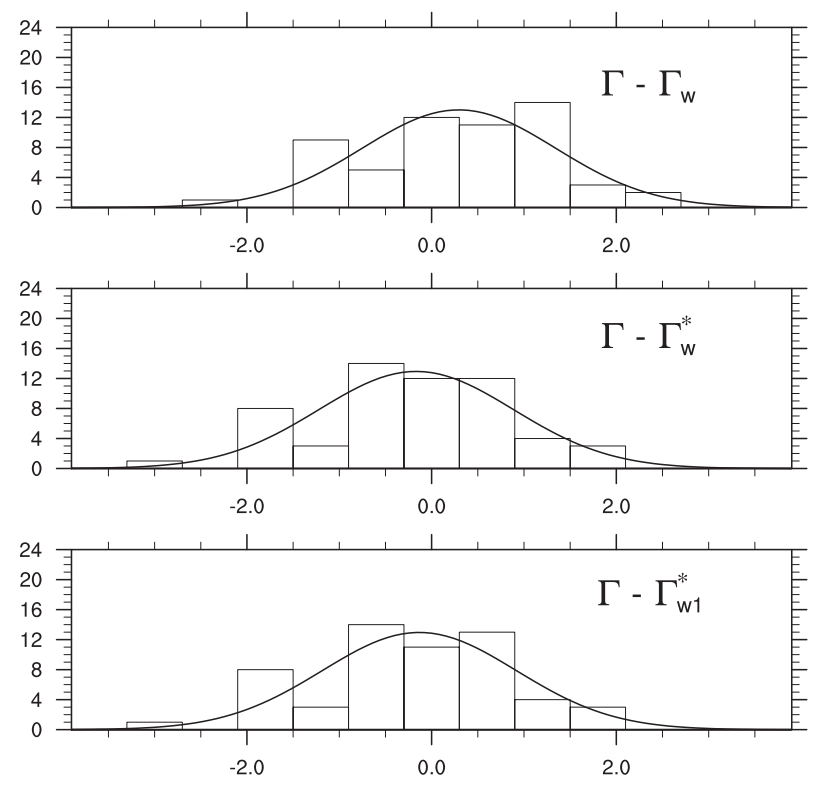

FIG. 2. As in Fig. 1, but using 200-m layers (57 values) and with the $y$-axis range scaled to about 57:118.

gave similar results, in spite of the different synoptic conditions and different mean LLJ wind speed. This result is consistent with the observation that the mean static stability of the pre-cold-frontal LLJ air masses approaching the coast in both CALJET and PACJET are similar, in spite of the different ENSO-modulated thermodynamic and kinematic characteristics found in these two experiments (Ralph et al. 2005).

As was previously pointed out, the $\Gamma-\Gamma_{w}$ distribution is characterized by such an abundance of data in the central bin ( $36 \%$ of the sample) that the number of values that fall in this bin differs significantly both from that of the other bins and from the number of values expected by a normal distribution using the observed mean and standard deviation (continuous curve in Fig. 1). This feature persists even if the analysis is performed on different subsets by grouping the data according to height, wind speed, or shear, but disappears, surprisingly, if the analysis is performed increasing the layer depth from 100 to $200 \mathrm{~m}$ and retaining only the layers that are composed by a pair of members of the $100-\mathrm{m}$ statistics (Fig. 2 and Table 2). This implies that many adiabatic layers are thinner than $200 \mathrm{~m}$, and therefore could be related to local processes like turbulent mixing.

This characteristic and the central peak of the $\Gamma-\Gamma_{w}$ distribution in Fig. 1 suggest the hypothesis that in the CALJET and PACJET experiments the lapse-rate statistics can be considered a (local) collection of saturated adiabatic lapse rates in a background normal distribution, which is representative of the (global) stability 
TABLE 2. As in Table 1, but using 200-m layers, with $n=57$.

\begin{tabular}{llll}
\hline & \multicolumn{1}{c}{$\overline{\Gamma-\Gamma_{w}}$} & $\overline{\Gamma-\Gamma_{w}^{*}}$ & $\overline{\Gamma-\Gamma_{w 1}^{*}}$ \\
\hline $\bar{d} \pm s_{\bar{d}}$ & $0.30 \pm 0.14$ & $-0.17 \pm 0.14$ & $-0.13 \pm 0.14$ \\
$z_{\bar{d}}=\bar{d} / s_{\bar{d}}$ & 2.14 & -1.21 & -0.93 \\
$P\left(|z|>\left|z_{\bar{d}}\right|\right)$ & $3.24 \%$ & $22.63 \%$ & $35.24 \%$ \\
\hline
\end{tabular}

conditions of the atmospheric rivers. To evaluate the mean value of this latter distribution, it is nevertheless necessary to discard the excess of adiabatic values. From a visual inspection of the $\Gamma-\Gamma_{w}$ distribution in Fig. 1, a reasonable choice seems that of discarding the shaded part of the central bin ( 27 values) by supposing that the normal distribution predicts in this bin the same number of values as in the adjacent right one (the mean value almost coincides with the right edge of the central bin). The discarded values in the $\Gamma-\Gamma_{w}$ distribution have their equivalents in the other two distributions of Fig. 1, but because of the above-mentioned mean shift of $0.46 \mathrm{~K} \mathrm{~km}^{-1}$ between $\overline{\Gamma_{w}^{*}}$ and $\overline{\Gamma_{w}}$ and of $0.43 \mathrm{~K} \mathrm{~km}^{-1}$ between $\overline{\Gamma_{w 1}^{*}}$ and $\overline{\Gamma_{w}}$, they split into two bins that cover the interval $(-0.9,+0.3) \mathrm{K} \mathrm{km}^{-1}$. A split of 21 and 6 , respectively, in the $\Gamma-\Gamma_{w}^{*}$ distribution and of 19 and 8 , respectively, in the $\Gamma-\Gamma_{w 1}^{*}$ distribution is obtained (shaded areas in Fig. 1). Mean values and standard deviations of the modified empirical distributions are showed in Table 3 and the corresponding curves of the expected values from the normal distribution have been added (dotted lines) in Fig. 1. The agreement between the modified distributions and the expected values is very good, and the previous decisions about the null hypothesis $H_{0}$ are strengthened, because the mean difference between the lapse rate and the saturated adiabatic lapse rate $\Gamma_{w}$ is increased [the result is highly significant, since $\left.P\left(|z|>\left|z_{\bar{d}}\right|\right)<1 \%\right]$ and both mean differences between the lapse rate and $\Gamma_{w}^{*}$ and $\Gamma_{w 1}^{*}$ are now almost equal to zero. The above-mentioned hypothesis about the lapse-rate distribution reinforces therefore the current opinion about the weak stability conditions of the atmospheric rivers by suggesting their neutrality.

\section{Conclusions}

The analysis of the temperature and humidity profiles in CALJET-1998 and PACJET-2001 experiments confirms that in order to assess the static stability of the lowest layers of a saturated atmosphere, the simple method of comparing the observed lapse rate with the expression of Richiardone and Giusti (2001) is equivalent to analyzing the sign of the expression of Durran and Klemp (1982) for the square of the Brunt-Väisälä frequency. Both
TABLE 3. As in Table 1, but after having discarded the 27 values from the central bin of the $\Gamma-\Gamma_{w}$ distribution (shaded areas in Fig. 1).

\begin{tabular}{llll}
\hline \hline & \multicolumn{1}{c}{$\overline{\Gamma-\Gamma_{w}}$} & $\overline{\Gamma-\Gamma_{w}^{*}}$ & $\overline{\Gamma-\Gamma_{w 1}^{*}}$ \\
\hline $\bar{d} \pm s_{\bar{d}}$ & $0.39 \pm 0.15$ & $-0.01 \pm 0.15$ & $0.00 \pm 0.15$ \\
$z_{\bar{d}}=\bar{d} / s_{\bar{d}}$ & 2.60 & -0.07 & 0.00 \\
$P\left(|z|>\left|z_{\bar{d}}\right|\right)$ & $0.03 \%$ & $94.42 \%$ & $100.00 \%$ \\
\hline
\end{tabular}

methods indicate that, on the mean, the CALJET-1998 and PACJET-2001 profiles are approximately neutral. In particular, the shape of the lapse-rate distribution supports the hypothesis that the lapse-rate statistics is a local collection of saturated adiabatic lapse rates in a background normal distribution centered around the neutrality.

On the contrary, the profiles would be classified, in the mean, as significantly unstable if the stability would be assessed by comparing the observed lapse rate with the saturated adiabatic one. It is therefore confirmed experimentally that it is not completely correct to assess the moist neutrality using this method.

Acknowledgments. The authors thank Richard Rotunno, Paul J. Neiman, and two anonymous reviewers for their comments on the manuscript.

\section{REFERENCES}

Browning, K. A., and C. W. Pardoe, 1973: Structure of low-level jet streams ahead of mid-latitude cold fronts. Quart. J. Roy. Meteor. Soc., 99, 619-638.

Durran, D., and J. B. Klemp, 1982: On the effects of moisture on the Brunt-Väisälä frequency. J. Atmos. Sci., 39, 21522158.

Green, J. R., and D. Margerison, 1978: Statistical Treatment of Experimental Data. Elsevier Scientific, 382 pp.

Lalas, D. P., and F. Einaudi, 1974: On the correct use of the wet adiabatic lapse rate in stability criteria. J. Appl. Meteor., 13, 318-324.

Ralph, F. M., P. J. Neiman, G. A. Wick, and C. S. Welden, 2004: Satellite and CALJET aircraft observations of atmospheric rivers over the eastern North Pacific Ocean during the winter of 1997/98. Mon. Wea. Rev., 132, 1721-1745.

_ _ - - and R. Rotunno, 2005: Dropsonde observations in low-level jets over the northeastern Pacific Ocean from CALJET-1998 and PACJET-2001: Mean vertical-profile and atmospheric-river characteristics. Mon. Wea. Rev., 133, 889-910.

Richiardone, R., and F. Giusti, 2001: On the stability criterion in a saturated atmosphere. J. Atmos. Sci., 58, 2013-2017.

Taylor, J., 1997: An Introduction to Error Analysis: The Study of Uncertainties in Physical Measurements. 2nd ed. University Science Books, 327 pp.

Zhu, Y., and R. E. Newell, 1998: A proposed algorithm for moisture fluxes from atmospheric rivers. Mon. Wea. Rev., 126, $725-735$. 